\title{
Diastolic dyssynchrony and its exercise-induced changes affect exercise capacity in patients with heart failure with reduced ejection fraction
}

\author{
Jakub Stępniewski, Grzegorz Kopeć, Wojciech Magoń, Piotr Podolec \\ Department of Cardiac and Vascular Diseases, Jagiellonian University Medical College, \\ John Paul II Hospital, Krakow, Poland
}

\begin{abstract}
Background: Left ventricular diastolic dyssynchrony is common in patients with heart failure with reduced ejection fraction (HFREF). Little is known however, about its pathophysiology and clinical effects. Herein is hypothesized that presence of diastolic dyssynchrony at rest or at exercise may importantly contribute to HF symptoms. The aim was to investigate the influence of diastolic dyssynchrony and its exercise-induced changes on exercise capacity in HFREF patients.

Methods: Patients with stable, chronic HF, left ventricular ejection fraction $<35 \%$, sinus rhythm and $Q R S \geq 120 \mathrm{~ms}$ were eligible for the study. Rest and cyclo-ergometer exercise echocardiography were performed. Diastolic dyssynchrony was defined as opposing-wall-diastolic-delay $\geq 55$ ms measured in tissue-Doppler imaging. Exercise capacity was assessed by peak oxygen consumption $\left(V_{2 p e a k}\right)$. Association between diastolic dyssynchrony and $V_{2 \text { peak }}$ was assessed in univariate regression analysis and further adjusted for possible confounders.

Results: Fourty eight patients were included (aged $63.7 \pm 12.2)$. Twenty-seven (56.25\%) had diastolic dyssynchrony at rest and 13 (27\%) at exercise. Twenty-two (46\%) experienced a change in diastolic dyssynchrony status during exercise. In univariate models diastolic dyssynchrony at rest or at exercise were associated with lower $V_{2 \text { peak }}$ (beta coefficient $=-3.8, p=0.004$; beta coefficient $=-3.6, p=0.02$, respectively). However, the ability to restore diastolic synchronicity during exercise was associated with higher $V O_{2 p e a k}$ (beta coefficient $=3.4, p=0.04$ ) and remained an important predictor of exercise capacity after adjustment for age and HF etiology.

Conclusions: The ability to restore diastolic synchronicity at exercise predicts exercise capacity in patients with HFREF. (Cardiol J 2021; 28, 6: 932-940)

Key words: stress echocardiography, cardiopulmonary exercise test, ischemic cardiomyopathy, QRS prolongation, cardiac resynchronization therapy
\end{abstract}

\section{Introduction}

Mechanical dyssynchrony results from the incoordinate wall motion of different ventricular segments. It may occur not only in systole, but also in diastole. While systolic dyssynchrony has been shown to be an important contributor to the left ventricular (LV) dysfunction in a wide spectrum of heart failure (HF) patients, little is known on the pathophysiology and clinical effects of diastolic dyssynchrony, although diastolic dyssynchrony has been frequently observed both in HF patients with reduced (HFREF) and preserved (HFPEF) ejection fraction [1-5].

Most evidence regarding diastolic dyssynchrony come from studies on patients with HFPEF.

Address for correspondence: Jakub Stępniewski, MD, Department of Cardiac and Vascular Diseases, Jagiellonian University Medical College, John Paul II Hospital, ul. Prądnicka 80, 31-202 Kraków, Poland, tel: +48 1261422 87, fax: +48 1242343 76, e-mail: jakub.stepniewski@gmail.com

Received: 2.01.2019 Accepted: 5.02.2019 Early publication date: 26.03.2019

This article is available in open access under Creative Common Attribution-Non-Commercial-No Derivatives 4.0 International (CC BY-NC-ND 4.0) license, allowing to download articles and share them with others as long as they credit the authors and the publisher, but without permission to change them in any way or use them commercially. 
Diastolic dyssynchrony in this population was found to aggravate LV diastolic dysfunction [5]. It was associated with the LV hypertrophy and increased LV mass [5]. Interestingly, initiation of medical therapy has shown to favor improvement of diastolic dyssynchorny. Patients with HFPEF, in whom treatment with diuretics, when beta-blockers and angiotensin-converting enzyme inhibitors or angiotensin receptor antagonists were initiated, experienced a significant decrease in diastolic dyssynchrony [5].

In contrast, an understanding of the pathophysiology and clinical effects of diastolic dyssynchrony in HFREF patients remains deficient. It was found, that diastolic dyssynchrony occurs at least as frequent as systolic, although the coexistence of systolic and diastolic dyssynchrony is low, suggesting that the mechanisms of these two phenomena may exhibit some differences [3]. The presence of diastolic dyssynchrony was observed more often in patients with wider QRS duration and was linked to worse diastolic LV function [1]. It was also associated with an adverse prognosis in children with dilated cardiomyopathy [6]. Eventually, cardiac resynchronization therapy (CRT) was found to improve diastolic dyssynchrony, however the role of diastolic dyssynchrony in CRT patient selection is less clear [3].

Data on the influence of diastolic dyssynchrony on exercise capacity in patients with HFREF is lacking. It was also unknown as to whether diastolic dyssynchrony is prone to change during exercise as had previously been reported with regard to systolic dyssynchrony [7].

Limitation of exercise capacity is the main symptom of HF, hence the aim in the present study to investigate the influence of diastolic dyssynchrony on exercise capacity in HFREF patients with prolonged QRS duration, and to analyse the effect of exercise on the presence of LV diastolic dyssynchrony.

\section{Methods}

\section{Study population}

Consecutive HFREF patients considered for CRT device implantation at a single tertiary cardiology department between 2013 and 2014 were included in this study. Patients were enrolled in the study if they classified in the New York Heart Association (NYHA) functional class II-IV despite optimal medical therapy and had no further coronary revascularization options, presented with LV ejection fraction (LVEF) $\leq 35 \%$, were in sinus rhythm and had QRS duration $\geq$ $\geq 120 \mathrm{~ms}$ on a 12 -lead electrocardiogram. Patents with any HF exacerbations within past 3 months were not eligible for the study. Exclusion criteria also included a history of any cardiac implantable electronic devices, persistent atrial fibrillation, significant respiratory, neurological or orthopaedic disorder limiting exercise capabilities. Ischemic etiology (ischemic cardiomyopathy [ICM]) of the HF was defined based on a history of myocardial infarction, coronary revascularization or presence of angiographically significant stenotic coronary lesions. Otherwise the patients were diagnosed with non-ischemic cardiomyopathy (DCM).

All patients provided written informed consent to participate in this study. The study was performed in accordance with the Declaration of Helsinki and was approved by the Institutional Ethical Committee at the Jagiellonian University in Krakow, Poland (KBET/110/B/2013).

All measurements and patient medical records were prospectively acquired by the authors themselves.

\section{Echocardiography}

A Vivid 7 device (GE Medical System, Horten, Norway) was used, equipped with phased-array 3.5-MHz transducer and tissue Doppler imaging (TDI) software to perform echocardiographic examinations in all patients. The recordings were analysed offline on EchoPac software (GE Vingmed, Horten, Norway) after digital storage. Conventional 2-dimensional and Doppler parameters were calculated in apical 4-, 3- and 2-chamber views. TDI data were recorded with the highest attainable frame rate. Six basal LV time-velocity curves (TVI) were reproduced offline from stored TDI color images to analyse LV systolic and diastolic dyssynchrony. All measurements were performed by experienced echocardiographers by averaging at least 3 consecutive heart beats.

The LV systolic function was determined by LVEF, calculated using the Simpson biplane method [8]. The LV diastolic function was quantified by the ratio of the early diastolic mitral velocity (E) acquired with the pulsed-wave Doppler, to the mean septal and lateral mitral annulus early diastolic velocity (e') obtained by the TDI-derived pulsedwave (E/e') [9].

\section{Exercise stress echocardiography}

Symptom-limited exercise echocardiography was performed on cycle ergometer (Ergoline 9000 Ergoline GmbH, Bitz, Germany) in a semi-recum- 


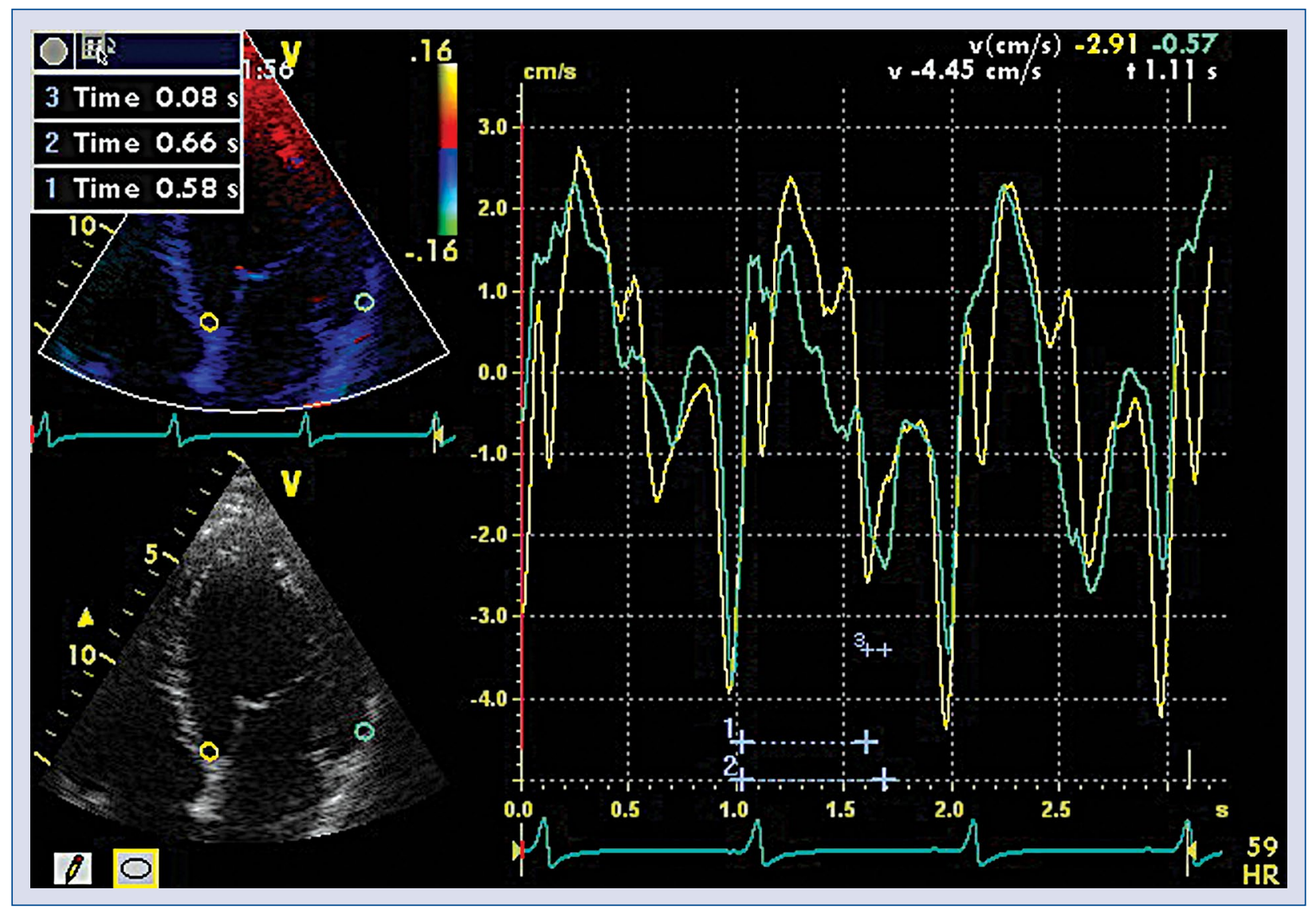

Figure 1. An example of diastolic dyssynchrony evaluation on the myocardial velocity imaging curves. The yellow curve represents basal segment of the interventricular septum (IVS) and the green, a basal segment of the lateral wall (LAT). Line 1 and 2 measure the time-to-peak early diastolic myocardial velocity interval (Te) of the IVS (line 1) and the LAT (line 2) from the onset of the QRS. Line 3 shows the difference between the IVS Te and the LAT Te representing an opposing wall Te delay.

bent position with the initial workload of 20 Watts increasing every 2 min by 20 Watts. Echocardiographic data were recorded throughout the exercise and stored for subsequent off-line analysis. Peak exercise measurements were derived from at least 3 heart beats in the last 2-min cycle and are expressed as means. Pharmacotherapy was continued without change at the time of stress tests.

\section{Diastolic and systolic LV dyssynchrony}

Calculations of the LV diastolic and systolic dyssynchrony were performed at rest and at peak exercise for each patient. $6 \mathrm{LV}$ basal TVIs were applied to measure time-to-peak early diastolic myocardial velocity intervals (Te) from the onset of QRS complex to the peak early diastolic myocardial velocity to analyse diastolic dyssynchrony (Fig. 1), and time-to-peak systolic velocity intervals (Ts) from the onset of QRS complex to the peak myocardial systolic velocity in order to analyse systolic dyssynchrony. The differences between Te and Ts of opposing wall segments were calculated to determine opposing wall diastolic and systolic delays. In order to eliminate the effect of heart rate on measurements of dyssynchrony parameters and to allow for comparison of rest and exercise dyssynchrony status, Te and Ts delays were corrected for RR intervals using the Bazzet formula [10]. At least one corrected opposing wall diastolic delay greater or equal to $55 \mathrm{~ms}$ was indicative for rest and exercise diastolic dyssynchrony, as previously described [3]. Similarly, at least one corrected opposing wall systolic delay greater or equal to $65 \mathrm{~ms}$ was indicative for rest an exercise systolic dyssynchrony [11].

Exercise-induced diastolic resynchronization was defined as change from the presence of diastolic dyssynchrony at rest to absence of diastolic dyssynchrony at exercise. In contrast, occurrence of diastolic dyssynchrony during exercise if not 
present at rest was indicative of exercise-induced diastolic dyssynchronization.

\section{Cardiopulmonary exercise test}

The treadmill cardiopulmonary exercise test (CPET) was employed to determine exercise capacity. The tests were conducted on a separate day than stress echocardiography. The CPET was performed using Reynolds Medical TMX425 TRACKMASTER treadmill unit with continuous breath-by-breath measurement of oxygen consumption $\left(\mathrm{VO}_{2}\right)$, carbon dioxide production $\left(\mathrm{VCO}_{2}\right)$, and minute ventilation (VE) on Reynolds Medical ZAN-600 respiratory gas analyzer. Modified Naughton protocol was applied in all patients [12]. $\mathrm{VO}_{2 \text { peak }}$ was defined as the highest value of oxygen uptake attained in the final $30 \mathrm{~s}$ of exercise and was presented as a weighted variable $(\mathrm{mL} / \mathrm{kg} /$ $/ \mathrm{min}$ ) and as a percentage of age and sex predicted maximal exercise oxygen consumption. Anaerobic threshold was defined as the submaximal $\mathrm{VO}_{2}$ level when there is a dislinear rise in $\mathrm{VE}$ and $\mathrm{VCO}_{2}$ and expressed as $\mathrm{mL} / \mathrm{kg} / \mathrm{min}$. Respiratory exchange ratio was calculated as the $\mathrm{VCO}_{2} / \mathrm{VO}_{2}$.

\section{Statistical analysis}

Categorical variables were described as counts and percentages and continuous variables as means \pm standard deviations or median and interquartile range. the unpaired Student's t-test was used for normally distributed variables, the Mann-Whitney U-test for non-normally distributed continuous data, and the $\chi^{2}$ test for categorical data to compare patients with and without diastolic dyssynchrony. With the use of univariate regression analysis the association between $\mathrm{VO}_{2 \text { peak }}$ (dependent variable) were examined and its potential modifiers (independent variables) including age, sex $(0-$ male, 1 - female), HF etiology ( 0 - DCM, 1 - ICM), serum hemoglobin level, PR interval, QRS duration and morphology $(0-$ non-left bundle branch block [LBBB], 1 - LBBB), markers of the LV systolic $(\mathrm{LVEF})$ and diastolic function (E/e' ratio), the presence of rest and exercise diastolic and systolic dyssynchrony $(0-$ not present, $1-$ present $)$ and the presence of exercise-induced diastolic resynchronization and exercise-induced dyssynchronization. Significant associations were further adjusted the for age and etiology. The significance level was set at $\mathrm{p}<0.05$. Statistical analyses were performed with Statistica PL software (StatSoft, Inc. 2017, STATISTICA, data analysis software system, version 13.1; www.statsoft.com) and MedCalc version 11.6.1.0 (MedCalc Software, Mariakerke, Belgium).

\section{Results}

\section{Characteristics of the patients studied}

Between 2013 and 2014 we recruited 54 patients. Further analyses were based on 48 patients aged $63.7 \pm 12.2(39$; $81.3 \%$ males $)$ with a sufficient quality of echocardiographic data. The majority of patients (30; 62.5\%) were in NYHA class III. Medical treatment of the patients studied complied with contemporary guidelines [13]. A summary of clinical characteristics of the study group is presented in Table 1 .

\section{Rest and stress echocardiography}

The mean LVEF was reduced $(23.6 \pm 6 \%)$ and the LV diastolic function was decreased with the mean $\mathrm{E} / \mathrm{e}$ ' ratio of $17.1 \pm 8$.

Exercise cardiac echo studies were performed without significant adverse events. Stress examinations were terminated at the mean workload of $76.2 \pm 30.5$ Watts and the mean heart rate of $115.4 \pm 22.1 \mathrm{bpm}$. Echocardiographic parameters are presented in Table 1.

\section{Diastolic LV dyssynchrony at rest and at exercise}

Diastolic dyssynchrony at rest was identified in $27(56.3 \%)$ patients, mainly in males $(25 ; 93 \%)$ and patients with ICM were (17;63\%). The group with diastolic dyssynchrony compared to the group without had similar LV systolic and diastolic function and a similar proportion of patients with systolic dyssynchrony. A detailed comparison of clinical parameters between patients with and without diastolic dyssynchrony is summarized in Table 1.

Exercise echocardiography revealed the presence of exercise diastolic dyssynchrony in 13 (27\%) patients. Twenty-two (46\%) patients experienced a change in the diastolic dyssynchrony status under exercise. Exercise-induced diastolic resynchronization was present in $18(66.6 \%)$ patients and provoked diastolic dyssynchrony in 4 (19\%) patients (Fig. 2). A detailed comparison of clinical parameters between patients who remained synchronized during exercise or became dyssynchronized and between patients who remained dyssynchronized or resynchronized during exercise is presented in Supplementary Table 1.

\section{Diastolic dyssynchrony and exercise capacity}

The CPET was performed in every patient but the full protocol was obtained in $41(85 \%)$. 
Table 1. Characteristics of the patients studied.

\begin{tabular}{|c|c|c|c|c|}
\hline Variable & $\begin{array}{l}\text { All patients } \\
\quad(n=48)\end{array}$ & $\begin{array}{l}\text { Without diastolic } \\
\text { dyssynchrony } \\
(n=21)\end{array}$ & $\begin{array}{l}\text { With diastolic } \\
\text { dyssynchrony } \\
(n=27)\end{array}$ & $\mathbf{P}$ \\
\hline Age [years] & $63.7 \pm 12.2$ & $63.5 \pm 9.6$ & $63.8 \pm 14$ & 0.93 \\
\hline Women/men & $9(18.7 \%) / 39(81.3 \%)$ & $7(33 \%) / 14(67 \%)$ & $2(7 \%) / 25(93 \%)$ & 0.02 \\
\hline Body mass index $\left[\mathrm{kg} / \mathrm{m}^{2}\right]$ & $26.5 \pm 3.8$ & $25.9 \pm 4.2$ & $26.9 \pm 3.5$ & 0.37 \\
\hline Ischemic/non-ischemic & $23(47.9 \%) / 25(52.1 \%)$ & $6(29 \%) / 15(71 \%)$ & $17(63 \%) / 10(37 \%)$ & 0.02 \\
\hline NYHA: & & & & 0.58 \\
\hline II & $12(25 \%)$ & $6(29 \%)$ & $6(22 \%)$ & \\
\hline III & $30(62.5 \%)$ & $13(62 \%)$ & $17(63 \%)$ & \\
\hline IV & $6(12.5 \%)$ & $2(9 \%)$ & $4(15 \%)$ & \\
\hline NT-proBNP [pg/mL] & 1667 [503-3309] & 1206 [532-2665] & 1744 [479-4888] & 0.47 \\
\hline Hemoglobin $[\mathrm{g} / \mathrm{dL}]$ & $14.4 \pm 1.2$ & $14.4 \pm 1$ & $14.3 \pm 1.3$ & 0.7 \\
\hline Heart rate $[\mathrm{bpm}]$ & $70.6 \pm 8.9$ & $71.2 \pm 8.8$ & $70,1 \pm 9.1$ & 0.69 \\
\hline QRS duration [ms] & 150 [120-160] & 160 [120-160] & 140 [120-160] & 0.51 \\
\hline $\mathrm{PR}[\mathrm{ms}]$ & 200 [160-220] & 180 [160-200] & 200 [160-220] & 0.08 \\
\hline LBBB/non-LBBB & $28(58.3 \%) / 20(41.7 \%)$ & $11(52 \%) / 10(48 \%)$ & $17(63 \%) / 10(37 \%)$ & 0.46 \\
\hline Beta-blocker & $47(97.9 \%)$ & $21(100 \%)$ & $26(96 \%)$ & 0.37 \\
\hline ACEI or ARB & $47(97.9 \%)$ & $20(96 \%)$ & $27(100 \%)$ & 0.25 \\
\hline Aldosterone receptor antagonist & $43(89.6 \%)$ & $18(86 \%)$ & $25(93 \%)$ & 0.44 \\
\hline Loop diuretics & $44(91.6 \%)$ & $18(86 \%)$ & $26(96 \%)$ & 0.18 \\
\hline LVEF [\%] & $23.6 \pm 6.0$ & $23.5 \pm 6.6$ & $23.7 \pm 5.7$ & 0.91 \\
\hline $\mathrm{E} / \mathrm{e}^{\prime}$ ratio & $17.1 \pm 8.1$ & $15.3 \pm 6.2$ & $18.5 \pm 9.2$ & 0.17 \\
\hline Max Te delay [ms] & $60[40-80]$ & $40[30-45]$ & 80 [60-98] & $<0.001$ \\
\hline Systolic dyssynchrony & $32(66.6 \%)$ & $15(71 \%)$ & $17(63 \%)$ & 0.54 \\
\hline
\end{tabular}

NYHA - New York Heart Association; NT-proBNP - N-terminal prohormone of B-type natriuretic peptide; LBBB - left bundle branch block; ACEI - angiotensin converting enzyme inhibitor; ARB - angiotensin receptor blocker; LVEF — left ventricular ejection fraction; E/e' - ratio of early diastolic mitral velocity to early diastolic velocity of the mitral annulus; max Te delay - maximal opposing wall diastolic delay

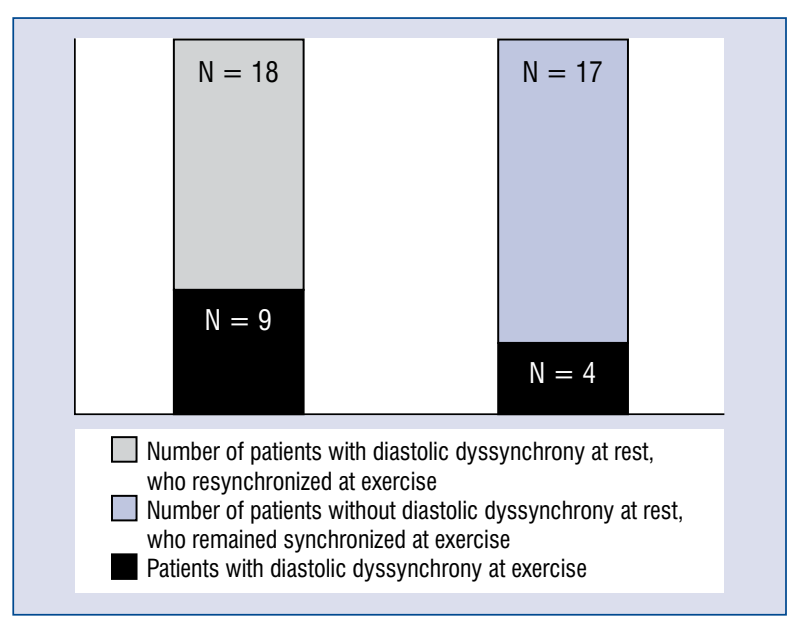

Figure 2. Exercise related changes of diastolic dyssynchrony. The first column shows proportion of patients with diastolic dyssynchrony at rest, who restored synchronicity at exercise (gray square) and those, who remained dyssynchronized (black square); The second column shows proportion of patients without diastolic dyssynchrony at rest, who remained synchronized at exercise (blue square) and those who dyssynchronized (black square).
Seven patients refused to complete the test due to anxiety for exercising on the treadmill or lightheadedness. Detailed CPET results are shown in Table 2. Lower $\mathrm{VO}_{\text {2peak }}$ was associated with more advanced age, ischemic etiology of HF, longer PR interval, higher $\mathrm{E} / \mathrm{e}$ ' ratio, the presence of rest and exercise diastolic dyssynchrony and lack of diastolic resynchronization at exercise (Table 3). After adjustment for age and etiology, exercise induced diastolic resynchronization (beta coefficient $=3.4$; $95 \%$ confidence interval $[\mathrm{CI}] 0.17$ to $6.6 ; \mathrm{p}=0.04$ ), $\mathrm{E} / \mathrm{e}$ ' (beta coefficient $=-2.0 ; 95 \% \mathrm{CI}-0.06$ to $-0.34 ; \mathrm{p}=0.006$ ) and PR interval (beta coefficient $=-0.05 ; 95 \%$ CI -0.02 to $-0.08, \mathrm{p}=0.004$ ) were significantly associated with $\mathrm{VO}_{2 \text { peak }}$ (Table 3 ). A comparison of mean $\mathrm{VO}_{2 \text { peak }}$ between patients without rest diastolic dyssynchrony, who remained synchronized at exercise and those who dyssynchronized at exercise, and between patients with rest diastolic dyssynchrony, who remained dyssnchronized at exercise and those who resynchronized at exercise is shown in Figure 3. 
Table 2. Cardiopulmonary exercise tests parameters.

\begin{tabular}{lcccc}
\hline Variable & $\begin{array}{c}\text { All patients } \\
(\mathbf{n}=\mathbf{4 1})\end{array}$ & $\begin{array}{c}\text { Without diastolic } \\
\text { dyssynchrony } \\
(\mathbf{n}=\mathbf{1 7})\end{array}$ & $\begin{array}{c}\text { With diastolic } \\
\text { dyssynchrony } \\
\text { (n = 24) }\end{array}$ & P \\
\hline Time of exercise [s] & $555 \pm 234$ & $661 \pm 247$ & $510 \pm 218$ & 0.14 \\
Exercise load [METs] & $5.4[4.4-6.3]$ & $5.4[4.4-7.4]$ & $4.7[3.4-5.4]$ & 0.16 \\
$\mathrm{VO}_{2 \text { peak }}[\mathrm{mL} / \mathrm{kg} / \mathrm{min}]$ & $15.5 \pm 4.3$ & $17.8 \pm 4$ & $13.9 \pm 4$ & 0.004 \\
Percentage of predicted $\mathrm{VO}_{2 \max }[\%]$ & $57.9 \pm 18.6$ & $65.4 \pm 23.5$ & $52.4 \pm 11.6$ & 0.04 \\
Anaerobic threshold $[\mathrm{mL} / \mathrm{kg} / \mathrm{min}]$ & $11[7.1-14.5]$ & $11.7[8.9-16.7]$ & $7.8[6.9-12.1]$ & 0.14 \\
VE/VCO & $31[27.3-37]$ & $28[25.1-30.4]$ & $33.3[30.1-39.5]$ & $<0.001$ \\
Respiratory exchange $\mathrm{ratio}$ & $1.03[0.96-1.12]$ & $1.03[0.96-1.09]$ & $1.03[0.95-1.14]$ & 0.62 \\
\hline
\end{tabular}

$\mathrm{VO}_{2 \text { peak }}$ - peak oxygen uptake; $\mathrm{VO}_{2 \max }$ - maximal exercise oxygen consumption; $\mathrm{VE} / \mathrm{VCO}_{2}-$ minute ventilation to carbon dioxide production ratio

Table 3. Associations between clinical, electro- and echocardiographic variables and $\mathrm{VO}_{2 \text { peak}}$.

\begin{tabular}{|c|c|c|c|c|}
\hline \multirow[t]{2}{*}{ Variable } & \multicolumn{2}{|c|}{ Univariate analysis } & \multicolumn{2}{|c|}{$\begin{array}{l}\text { Associations after adjustment } \\
\text { for age and etiology }\end{array}$} \\
\hline & $\begin{array}{c}\text { Beta coefficient } \\
(95 \% \mathrm{CI})\end{array}$ & $\mathbf{P}$ & $\begin{array}{c}\text { Beta coefficient } \\
(95 \% \mathrm{Cl})\end{array}$ & $\mathbf{P}$ \\
\hline Age [years] & $-0.17(-0.3$ to 0.05$)$ & 0.006 & & \\
\hline Etiology [0 - non-ischemic; $1-$ ischemic] & $-4.7(-6.8$ to -2.1$)$ & 0.0005 & & \\
\hline PR interval [ms] & $-0.06(-0.1$ to -0.03$)$ & 0.0013 & $-0.05(-0.08$ to -0.02$)$ & 0.004 \\
\hline QRS duration [ms] & $0.05(-0.01$ to 0.12$)$ & 0.1 & & \\
\hline LBBB [0 - absent; 1 - present] & $0.4(-2.4$ to 3.2$)$ & 0.78 & & \\
\hline LVEF [\%] & $0.09(-0.13$ to 0.32$)$ & 0.38 & & \\
\hline$E / e^{\prime}$ & $-0.27(-0.4$ to -0.12$)$ & 0.0006 & $-2.0(-0.34$ to -0.06$)$ & 0.006 \\
\hline Rest diastolic dyssynchrony & $-3.8(-6.35$ to -1.3$)$ & 0.004 & & \\
\hline Exercise diastolic dyssynchrony & $-3.6(-6.6$ to -0.6$)$ & 0.02 & & \\
\hline Exercise-induced diastolic resynchronization & $3.4(0.2$ to 6.6$)$ & 0.04 & $3.4(0.17$ to 6.6$)$ & 0.04 \\
\hline Rest systolic dyssynchrony & $1.5(-1.3$ to 4.4$)$ & 0.28 & & \\
\hline
\end{tabular}

$\mathrm{VO}_{2 \text { peak }}$ - peak oxygen consumption; LBBB — left bundle branch block; LVEF — left ventricular ejection fraction; E/e' — ratio of early diastolic mitral velocity to early diastolic velocity of the mitral annulus

\section{Discussion}

There are two major findings in this study. First, diastolic dyssynchrony may change during exercise. Some patients with diastolic dyssynchrony at rest experienced diastolic resynchronization during exercise, while some of those without diastolic dyssynchrony at rest, became dyssynchroneous at exercise. Although such dynamic features have previously been attributed to systolic dyssynchrony in patients with HFREF $[7,14]$. According to available research this is the first report on the dynamic nature of diastolic dyssynchrony.

The presence of diastolic dyssynchrony was also found, both at rest and exercise, was associ- ated with decreased exercise capacity in HFREF patients, but diastolic resynchronisation during exercise improved this.

Similarly, a negative effect of diastolic dyssynchrony on exercise capacity was reported in hypertensive patients with LV hypertrophy. In this group of patients diastolic dyssynchrony was also associated with decreased exercise capacity [15].

Impaired LV synchronicity may occur not only during ventricular contraction, but also during ventricular relaxation. In fact, diastolic dyssynchrony was found to be at least as frequent as systolic. The prevalence of $\mathrm{LV}$ diastolic dyssynchrony in patients with HFREF and prolonged QRS duration ranged from $52 \%$ to $73 \%$ as compared to systolic (46-73\%) 


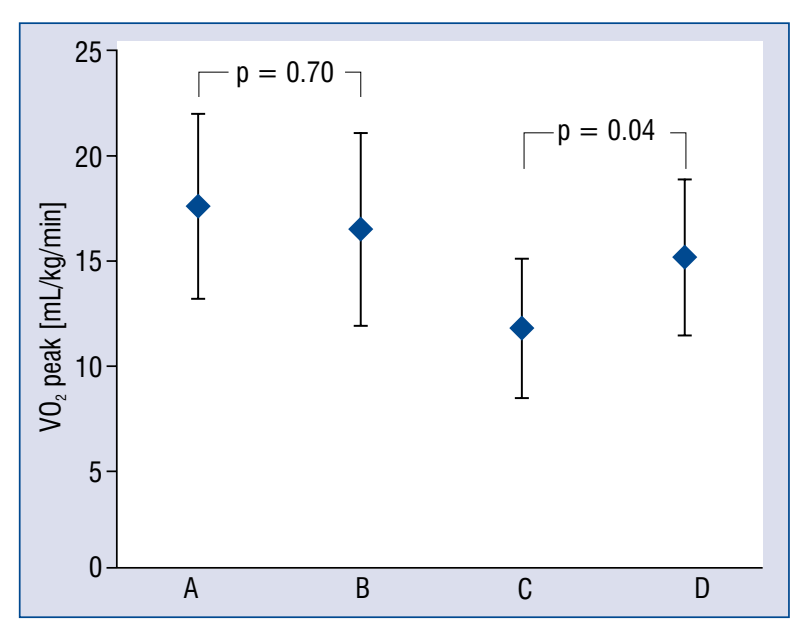

Figure 3. Comparison of mean $\mathrm{VO}_{2 \text { peak }}$ between patients without rest diastolic dyssynchrony, who remained synchronized at exercise (A) and those who dyssynchronized at exercise $(B)$; and between patients with rest diastolic dyssynchrony, who remained dyssnchronized at exercise (C) and those who resynchronized at exercise (D).

[1-3]. In the present study diastolic dyssynchrony was present in more than a half of the patients. A concurrent presence of systolic and diastolic dyssynchrony in the current study was seen in $35 \%$ of patients. A similar coincidence was shown in previous observations $[1,2]$. This would suggest that systolic and diastolic dyssycnhrony may have some different pathophysiologies.

Intraventricular diastolic dyssynchrony reflects inhomogeneous timing of relaxation in different myocardial segments [16]. In healthy hearts, all LV segments relax in an organized fashion producing suction forces at the most efficient energy consumption level. This is achieved by synchronous interplay between uninterrupted stimulation and undisturbed regional relaxability of cardiac myocytes. In the presence of diastolic dyssynchrony, relaxation of the LV becomes inefficient. Early diastolic discoordinate motion of the LV walls may cause impairment of the LV filling dynamics decreasing left atrial function, aggravating mitral regurgitation, adversely affecting right-to-left ventricular interaction and concomitantly hinder ejection properties [17, 18]. As cardiac output is dependent not only on systolic emptying but also on diastolic filling, diastolic dyssynchrony may cause additive hemodynamic compromise in a failing heart. Decreased global performance of the heart at an increased workload promotes consequently, in chronic unfavourable cardiac remodelling leading to a worsening of symptoms [19].
The causes of diastolic dyssynchrony has been less well understood. As systole and diastole are closely linked, it would be most expected that systolic asynchrony determined the presence of diastolic dyssynchrony. However, as discussed earlier, less than one-half of the HFREF patients have a coexistent systolic and diastolic dyssynchrony. This lack of concurrence may in part be attributed to the regional heterogeneity of the load-induced relaxation delay. It has been previously demonstrated in experimental studies, that global LV afterload translates to regional loading conditions in an ununiformed fashion [20,21]. It was shown, that regional myocardial load correlates with the timing of local relaxation [22]. Differences in regional loading conditions may thus result in regional discoordinate diastolic motion.

In relation to this, the LV diastolic dysfunction and its regional differences may also play a role in the pathophysiology of diastolic dyssynchrony. It has been shown, that diastolic dyssynchrony correlated with the degree of $L V$ diastolic dysfunction, prolonged relaxation and elevated pulmonary capillary wedge pressure in patients with HFPEF, but also in HFREF $[1,5,23]$. The present study found that, although not statistically significant, patients with diastolic dyssynchrony had higher estimated LV filling pressure than those without. Several studies have additionally proposed, that right-toleft ventricular diastolic interaction from raised right ventricular diastolic pressure could explain delayed onset of myocardial diastolic motion [24, 25]. Differences in serum fibrosis markers representing different LV diastolic dysfunction stages might thus help to indicate patient with advanced diastolic dyssynchrony [26].

Another potential circumstance for the occurrence of diastolic dyssynchrony may include myocardial ischemia. It the present study, it was observed that a majority of patients with diastolic dyssynchrony had ischemic origin of HF, whereas DCM was more prevalent among those without. Data from several studies on diastolic dyssynchrony in coronary artery disease come in line with the current observations. In 1 study diastolic dyssynchrony was evident in patients with ischemic heart disease and preserved ejection fraction and correlated with lower diastolic LV function $[27,28]$. In another, $\mathrm{LV}$ diastolic synchronicity was shown to be disrupted in patients with ST segment elevation myocardial infarction [29]. Interestingly, diastolic dyssynchrony recovered after successful coronary revascularization. Additionally, a study investigating the occurrence of diastolic dyssynchrony during 
dobutamine stress echocardiography found that diastolic dyssynchrony imaging at peak dobutamine yielded high sensitivity and specificity in predicting significant coronary artery disease [30]. This might imply, that diastolic dyssynchrony is related to the presence of viable myocardium and could partly explain dynamic changes of diastolic dyssynchrony observed in the present study. In contrast to the previously cited study, diastolic dyssynchrony has been shown to improve during dobutamine stress echocardiography in patients with LV hypertrophy, demonstrating that the lusitropic effect of dobutamine improve LV regional diastolic asynchrony led to an improvement of global LV diastolic filling [31]. Furthermore, CRT and medical therapy has been found to restore diastolic synchronicity. Wang et al. [5] who explored the effect of medical therapy on the extent of diastolic dyssynchrony in HF patients with preserved ejection fraction has shown, that the initiation of treatment with diuretics, beta-blockers and angiotensin-converting enzyme inhibitors or receptor blockers resulted in an improvement of diastolic dyssynchrony [5]. Accordingly, as wider QRS has been associated with the presence of diastolic dyssynchrony, it has been hypothesized, that CRT may also lead to diastolic resynchronization. Shanks et al. [3] observed a high incidence of diastolic dyssynchrony in CRT responders, and its sustained improvement with biventricular pacing. Although diastolic together with systolic dyssynchrony has been shown to normalise with CRT in some patients, it is questionable whether the presence of diastolic dyssynchorny improves CRT patient selection.

Diastolic dyssynchrony and its changes appear to have multifactorial pathophysiology, which include electrical and local mechanical properties of the myocardium, but the exact mechanism or clinical meaning remains uncertain. As discussed earlier, the presence of diastolic dyssynchrony may be attributed to myocardial ischemia or myocardial viability, as well as suboptimal medical therapy. Whether exercise-induced diastolic resynchronization could serve as a marker to guide therapy optimization requires further study.

\section{Limitations of the study}

The main strength of the present study is that it enriches a limited body of literature on the clinical implications of diastolic LV dyssynchrony and its exercise-induced changes in patients with HFREF and helps to improve our understanding of this phenomenon.
Despite these advantages there are several limitations. It was a pilot, single-center study with a limited number of participants. A limited number of LV dyssynchrony parameters were used. Measurement of dyssynchrony at peak exercise may pose some difficulties caused by insufficient quality of echocardiographic recordings in up to $10 \%$ of patients. There was no attempt to evaluate the causes of diastolic dyssynchrony and its exercise-induced changes. Despite these drawbacks, it is believed that the consistency of the results validates the observations. Larger scale prospective studies are needed to validate the present results.

\section{Conclusions}

Diastolic dyssynchrony may change during exercise in half of HFREF patients. The ability to restore diastolic synchronicity at exercise predicts better aerobic capacity, whereas advanced age, ischemic etiology, prolonged PR interval and more advanced diastolic dysfunction are associated with lower exercise tolerance.

\section{Conflict of interest: None declared}

\section{References}

1. Yu CM, Lin H, Zhang Q, et al. High prevalence of left ventricular systolic and diastolic asynchrony in patients with congestive heart failure and normal QRS duration. Heart. 2003; 89(1): 54-60, indexed in Pubmed: 12482792.

2. Schuster I, Habib G, Jego C, et al. Diastolic asynchrony is more frequent than systolic asynchrony in dilated cardiomyopathy and is less improved by cardiac resynchronization therapy. J Am Coll Cardiol. 2005; 46(12): 2250-2257, doi: 10.1016/j. jacc.2005.02.096, indexed in Pubmed: 16360054.

3. Shanks M, Bertini M, Delgado V, et al. Effect of biventricular pacing on diastolic dyssynchrony. J Am Coll Cardiol. 2010; 56(19): 1567-1575, doi: 10.1016/j.jacc.2010.01.077, indexed in Pubmed: 21029873.

4. Yu CM, Zhang Q, Yip GWK, et al. Diastolic and systolic asynchrony in patients with diastolic heart failure: a common but ignored condition. J Am Coll Cardiol. 2007; 49(1): 97-105, doi: 10.1016/j. jacc.2006.10.022, indexed in Pubmed: 17207728.

5. Wang J, Kurrelmeyer KM, Torre-Amione G, et al. Systolic and diastolic dyssynchrony in patients with diastolic heart failure and the effect of medical therapy. J Am Coll Cardiol. 2007; 49(1): 88-96, doi: 10.1016/j.jacc.2006.10.023, indexed in Pubmed: 17207727 .

6. Friedberg MK, Roche SL, Mohammed AF, et al. Left ventricular diastolic mechanical dyssynchrony and associated clinical outcomes in children with dilated cardiomyopathy. Circ Cardiovasc Imaging. 2008; 1(1): 50-57, doi: 10.1161/CIRCIMAGING. 108.782086, indexed in Pubmed: 19808514. 
7. Stępniewski J, Kopeć G, Magoń W, et al. Ischaemic aetiology predicts exercise dyssynchrony in patients with heart failure with reduced ejection fraction. Kardiol Pol. 2018; 76(10): 1450-1457, doi: 10.5603/KP.a2018.0148, indexed in Pubmed: 30067277.

8. Lang R, Badano L, Mor-Avi V, et al. Recommendations for Cardiac Chamber Quantification by Echocardiography in Adults: An Update from the American Society of Echocardiography and the European Association of Cardiovascular Imaging. Eur Heart J Cardiovasc Imaging. 2015; 16(3): 233-271, doi: 10.1093/ehjci/jev014.

9. Galderisi M, Henein MY, D'hooge J, et al. European Association of Echocardiography. Recommendations of the European Association of Echocardiography: how to use echo-Doppler in clinical trials: different modalities for different purposes. Eur J Echocardiogr. 2011; 12(5): 339-353, doi: 10.1093/ejechocard/ jer051, indexed in Pubmed: 21555455.

10. Stoylen A. Left Ventricular Mechanics During Exercise: A Doppler and Tissue Doppler Study. Eur J Echocardiogr. 2003; 4(4): 286-291, doi: 10.1016/s1525-2167(03)00008-8.

11. Gorcsan J, Abraham T, Agler DA, et al. American Society of Echocardiography Dyssynchrony Writing Group, American Society of Echocardiography Dyssynchrony Writing Group, Heart Rhythm Society. Echocardiography for cardiac resynchronization therapy: recommendations for performance and reporting: a report from the American Society of Echocardiography Dyssynchrony Writing Group endorsed by the Heart Rhythm Society. J Am Soc Echocardiogr. 2008; 21(3): 191-213, doi: 10.1016/j. echo.2008.01.003, indexed in Pubmed: 18314047.

12. Balady GJ, Arena R, Sietsema K, et al. American Heart Association Exercise, Cardiac Rehabilitation, and Prevention Committee of the Council on Clinical Cardiology, Council on Epidemiology and Prevention, Council on Peripheral Vascular Disease, Interdisciplinary Council on Quality of Care and Outcomes Research. Clinician's Guide to cardiopulmonary exercise testing in adults: a scientific statement from the American Heart Association. Circulation. 2010; 122(2): 191-225, doi: 10.1161/ CIR.0b013e3181e52e69, indexed in Pubmed: 20585013.

13. McMurray JJV, Adamopoulos S, Anker SD, et al. ESC Guidelines for the diagnosis and treatment of acute and chronic heart failure 2012: The Task Force for the Diagnosis and Treatment of Acute and Chronic Heart Failure 2012 of the European Society of Cardiology. Developed in collaboration with the Heart Failure Association (HFA) of the ESC. Eur Heart J. 2012; 33(14): 1787-1847, doi: 10.1093/eurheartj/ehs104, indexed in Pubmed: 22611136.

14. Lafitte S, Bordachar P, Lafitte M, et al. Dynamic ventricular dyssynchrony: an exercise-echocardiography study. J Am Coll Cardiol. 2006; 47(11): 2253-2259, doi: 10.1016/j.jacc.2005.11.087, indexed in Pubmed: 16750692.

15. Jung IH, Seo HS, Kim MJ. P569. Diastolic dyssynchrony is associated with exercise intolerance in hypertensive patients with left ventricular hypertrophy. Eur Heart J Cardiovasc Imaging. 2016; 17(suppl_2): ii102-ii109, doi: 10.1093/ehjci/jew248.002, indexed in Pubmed: 28415098.

16. Cheng A, Helm RH, Abraham TP. Pathophysiological mechanisms underlying ventricular dyssynchrony. Europace. 2009; 11 Suppl 5: v10-v14, doi: 10.1093/europace/eup272, indexed in Pubmed: 19861385.

17. Kasner M, Westermann D, Schultheiss HP, et al. Diastolic heart failure and LV dyssynchrony. Curr Pharm Biotechnol. 2012; 13(13): 2539-2544, indexed in Pubmed: 22280429.
18. Kass DA. An epidemic of dyssynchrony: but what does it mean? J Am Coll Cardiol. 2008; 51(1): 12-17, doi: 10.1016/j. jacc.2007.09.027, indexed in Pubmed: 18174030.

19. Zhang Q, Yu CM. Clinical implication of mechanical dyssynchrony in heart failure. J Cardiovasc Ultrasound. 2012; 20(3): 117-123, doi: 10.4250/jcu.2012.20.3.117, indexed in Pubmed: 23185653.

20. Miura T, Bhargava V, Guth BD, et al. Increased afterload intensifies asynchronous wall motion and impairs ventricular relaxation. J Appl Physiol (1985). 1993; 75(1): 389-396, doi: 10.1152/ jappl.1993.75.1.389, indexed in Pubmed: 8376290.

21. Yano M, Kohno M, Konishi M, et al. Influence of left ventricular regional nonuniformity on afterload-dependent relaxation in intact dogs. Am J Physiol. 1994; 267(1 Pt 2): H148-H154, doi: 10.1152/ ajpheart.1994.267.1.H148, indexed in Pubmed: 8048579.

22. Aoyagi T, Iizuka M, Takahashi T, et al. Wall motion asynchrony prolongs time constant of left ventricular relaxation. Am J Physiol. 1989; 257(3 Pt 2): H883-H890, doi: 10.1152/ ajpheart.1989.257.3.H883, indexed in Pubmed: 2782445.

23. Waggoner AD, Rovner A, de las Fuentes L, et al. Clinical outcomes after cardiac resynchronization therapy: importance of left ventricular diastolic function and origin of heart failure. J Am Soc Echocardiogr. 2006; 19(3): 307-313, doi: 10.1016/j. echo.2005.10.014, indexed in Pubmed: 16500494.

24. Bleeker GB, Mollema SA, Holman ER, et al. Left ventricular resynchronization is mandatory for response to cardiac resynchronization therapy: analysis in patients with echocardiographic evidence of left ventricular dyssynchrony at baseline. Circulation. 2007; 116(13): 1440-1448, doi: 10.1161/CIRCULATIONAHA.106.677005, indexed in Pubmed: 17785624.

25. Atherton J, Moore T, Lele S, et al. Diastolic ventricular interaction in chronic heart failure. Lancet. 1997; 349(9067): 1720-1724, doi: 10.1016/s0140-6736(96)05109-4.

26. Wiśniowska-Śmiałek S, Dziewięcka E, Holcman K, et al. Kinetics of selected serum markers of fibrosis in patients with dilated cardiomyopathy and different grades of diastolic dysfunction of the left ventricle. Cardiol J. 2018 [Epub ahead of print], doi: 10.5603/CJ.a2018.0143, indexed in Pubmed: 30484268.

27. Bonow RO, Vitale DF, Bacharach SL, et al. Asynchronous left ventricular regional function and impaired global diastolic filling in patients with coronary artery disease: reversal after coronary angioplasty. Circulation. 1985; 71(2): 297-307, indexed in Pubmed: 3155499.

28. Lee PW, Zhang Q, Yip GWK, et al. Left ventricular systolic and diastolic dyssynchrony in coronary artery disease with preserved ejection fraction. Clin Sci (Lond). 2009; 116(6): 521-529, doi: 10.1042/CS20080100, indexed in Pubmed: 18684109.

29. Turan B, Daşli T, Erkol A, et al. Diastolic dyssynchrony in acute ST segment elevation myocardial infarction and relationship with functional recovery of left ventricle. J Cardiovasc Ultrasound. 2016; 24(3): 208-214, doi: 10.4250/jcu.2016.24.3.208, indexed in Pubmed: 27721951.

30. Onishi T, Uematsu M, Watanabe T, et al. Objective interpretation of dobutamine stress echocardiography by diastolic dyssynchrony imaging: a practical approach. J Am Soc Echocardiogr. 2010; 23(10): 1103-1108, doi: 10.1016/j.echo.2010.06.031, indexed in Pubmed: 20719470.

31. Liu J, Murata K, Fujino T, et al. Effect of dobutamine on regional diastolic left ventricular asynchrony in patients with left ventricular hypertrophy. Circ J. 2003; 67(2): 119-124, indexed in Pubmed: 12547992. 